sible instances in which the order of $T$ is 8 . Hence we observe that the required group is the well known simple group of order 168.

It is known that there are abelian groups which cannot be groups of cogredient isomorphisms.* Suppose that the group of cogredient isomorphisms $H$ of a given group $G$ is Hamiltonian. Each of the operators of $G$ which corresponds to identity of $H$ is self-conjugate in $G$. Since the non-identity commutator of $H$ is some power of each one of a set of generators of $H$, all the operators of $G$ which correspond to it must also be self-conjugate in $G$. As this is clearly impossible, it follows that a Hamiltonian group cannot be a group of cogredient isomorphisms. In fact, the preceding arguments apply to all groups which contain an operator different from identity, which is some power of each one of a set of generating operators. Hence such a group cannot be the group of cogredient isomorphisms of any group whatsoever.

CORNELL UNIVERSITY, February, 1900.

\title{
LOBACHEVSKY'S GEOMETRY.
}

Nikolaj Iwanowitsch Lobatschefskij. Zwei Geometrische Abhandlungen aus dem Russischen übersetzt, mit Anmerkungen und mit einer Biographie des Verfassers von FRIEDRICH Engel. Leipzig, B. G. Teubner, 1899. 8vo. xvi +476 pp.

This volume is the first in the series of the "Urkunden zur Geschichte der Nichteuklidischen Geometrie" planned by Friedrich Engel and Paul Stäckel in continuation of their "Theorie der Parallellinien von Euklid bis auf Gauss." The present work on Lobachevsky has been undertaken by Engel, while a volume on the two Bolyais by Stäckel is soon to appear. All geometricians will welcome the opportunity thus afforded of becoming fully acquainted with the writings of these men, who share with Gauss the honor of being the first to break loose from the authority of Euclid and to show that the latter's axiom concerning parallel lines is not only incapable of proof but entirely unnecessary in a logical geometry which fully explains all facts of experience. The earlier works of Lobachevsky have been until now locked up in the original Russian and their contents

*Comptes rendus, vol. 130, 1900, p. 316. 
are known to the public mainly through later abstracts in French and German. Of these the best known is the small pamphlet published in Berlin in 1840 with the title "Geometrische Untersuchungen zur Theorie der Parallellinien ", and translated into English by Professor G. B. Halsted. This, though containing the outline of the author's theory, is too concise to do it justice. Professor Engel has rendered therefore a signal service to mathematicians in translating the two articles which form the first part of the present volume.

The titles of these articles are, in the German translation, "Ueber die Anfangsgründe der Geometrie" and "Neue Anfaingsgründe der Geometrie mit einer vollständigen Theorie der Parallellinien." Both articles appeared in publications of the Kasan University, the former in 1829-30, the latter in 1836. A footnote by Lobachevsky himself informs us that the first article is taken from one presented to the Section for Mathematical-Physical Science in $\mathbf{1 8 2 6}$ under the title, "Exposition succincte des principes de la Géométrie," but no copy of this earlier work is extant. The "Anfangsgründe" is thus the earliest of Lobachevsky's publications on this subject. It is comparatively short, only sixty-six pages in the translation, and correspondingly concise, the proofs of the earlier theorems not being given. It is to the "Neue Anfangsgründe" that one must turn for the best introduction to Lobachevsky's thought. We follow this in our review.

The first six chapters present a development of geometric theorems as far as these do not depend upon any theory of parallel lines, and cin be read with approval by the most orthodox believer in the euclidean geometry. Lobachevsky's order of development is however entirely new. With him the distinctive geometric property of bodies is that of contact. This idea we get through the senses, back of it we can not go ; in fact, it is impossible to define it fully in words. By means of this property he discusses various kinds of sections of a body, and derives finally the conceptions of surface, line, and point, from the ways in which bodies or sections of bodies may touch each other. The distance of two points is their relative position and is determined by the contact of two bodies in the two points, independently of the form of these bodies outside of their points of contact. Hence, for example, a pair of dividers may determine distance and the idea of distance is independent of the conception of a straight line. The sphere is therefore the simplest solid, the spherical surface the sim- 
plest surface. A plane is determined by the intersections of equal spheres described about two fixed points as centers, and any two of these equal spheres define a circle. A straight line is defined as a line which remains in coincidence with itself in all positions when two points are fixed, and it is shown that in any circle there exist such lines, the diameters. With these ideas as the fundamental ones, it is clear that the geometry on the sphere is as simple as the geometry on the plane, and it is not surprising to find Lobachevsky carrying the two side by side. In one respect, at least, the spherical geometry is the simpler, for it may be shown that the sum of the angles of a spherical triangle is greater than $\pi$. In the plane geometry one must content himself with the theorems that the sum of the angles of a triangle cannot exceed $\pi$, and that if the sum for one triangle is equal to $\pi$ the same is true of all triangles, while if the sum is less than $\pi$ it is variable, decreasing as the lengths of the sides increase. At the outset Lobachevsky assumes that space is infinite. $\mathrm{He}$ is therefore able to prove that planes and straight lines are infinite in extent, that two straight lines can intersect in not more than one point, and that two perpendiculars to the same straight line cannot intersect. $\mathrm{He}$ misses, therefore, the elliptic geometry in which the sum of the angles of a triangle is greater than $\pi$. This geometry exists for him of course upon the sphere, and the formulas which he obtains later for plane triangles are shown by him to include the spherical formulas, but the possibility of the existence of this geometry on the plane is explicitly denied. It remained for Riemann to take the last step in the freeing of geometry from unnecessary hypotheses.

In chapter VII. begins the discussion of parallel lines on which the fame of the author rests. An axiom of Euclid asserts that if a straight line intersect two straight lines forming two interior angles on the same side of the first straight line which are together less than two right angles, then the two straight lines if sufficiently produced will meet. Many attempte have been made to justify this axiom by showing that its denial involves contradictions; but all such attempts have failed. Lobachevsky boldly denies the truth of the axiom and asserts that all lines radiating from a point fall into two classes with reference to any fixed line; namely, the converging lines which meet the fixed line, and the diverging lines which do not meet it. To the latter class belong two parallel lines which form the boundary between the two classes. More precisely, if $C$ is a point at a perpendicular distance $\alpha$ from a line $A B$, then there exists 
a certain angle $I I(\alpha)$ such that all lines which make with the perpendicular on the side toward $A B$ an angle less than $11(a)$ will meet $A B$, while lines which make an angle equal or greater than $I I(a)$ will not meet $A B$. The two lines which make the angle $I(\alpha)$ with the perpendicular are the parallel lines, and the angle $\Pi(\alpha)$ is the angle of parallelism. If $\Pi(a)$ is identically equal to $\frac{1}{2} \pi$, the euclidean axiom results and it is readily shown that then the sum of the angles of a triangle equals $\pi$, and conversely. On the other hand, if the sum of the angles of a triangle is less than $\pi, \eta(\alpha)$ is variable, decreasing as $a$ increases, becoming $\frac{1}{2}$ only for $a=0$, and being equal to any given acute angle for some value of $a$. There result therefore two systems of geometry, the usual one, and the other, called rather unfortunately by Lobachevsky the "imaginary" geometry. In the ordinary geometrya circle approaches a straight line when its radius is indefinitely increased, and the revolution of a straight line about an axis perpendicular to it generates a plane. In the imaginary geometry the limit of a circle is a boundary curve characterized by the property that all normals are parallel to a fixed line and hence to each other. The revolution of a boundary curve about a normal generates a boundary surface, which is cut by any plane in either a boundary curve or a circle. If now a triangle is formed on a boundary surface by means of three boundary curves it may be shown that the sum of its angles equals $\pi$. This triangle plays in the imaginary geometry the rôle of the plane triangle in the usual geometry.

This closes the synthetic and more elementary part of Lobachevsky's work. He turns now to the analytic treatment of the imaginary geometry. The usual trigonometric formulas hold for a boundary triangle, and a point of attack is thus found for the determination of $I(a)$. It results that the angle of parallelism is an analytic function of its argument, expressed by the simple relation

$$
\tan \frac{1}{2} \Pi(x)=e^{-x}
$$

Formulas connecting the sides and angles of a triangle are next deduced. These formulas include those of the usual plane and spherical geometry, and are in fact exactly those which one obtains by writing the formulas of spherical trigonometry for a triangle with pure imaginary sides. To derive the formulas of the euclidean geometry it is only necessary to assume that the sides of the triangle are so small that all higher powers may be neglected. This is to 
assume that the world of our experience is very small in comparison with the unit of length used in the imaginary geometry.

The foregoing is a brief sketch of the prominent ideas of the "Neue Anfangsgründe." Turning back to the "Anfangsgründe" we find in addition a large number of applications to the determination of the lengths of curves, the areas of plane and curved surfaces, and the volumes of solids. Many readers who are already familiar with Lobachevsky's theory of parallels will find this part of the work of great interest; for it is not contained in any other of the author's works. There is also a discussion based on the parallax of fixed stars to show that the discrepancy between the usual and the imaginary geometry is too small to be detected experimentally.

The second part of the volume before us is occupied with notes and a life of Lobachevsky by the translator. Lobachevsky's argument is in its large outline simple and clear and the same is true in general of his separate theorems, but he is in places, obscure through too concise presentation or through suppression of intermediate reckoning. Occasionally also a demonstration is faulty. All such places have been carefully worked over by the annotator with the object and the result of making the work easily read by all. The notes are more copious in the case of the "Anfangsgründe" where the need is greater.

The biography is at the same time an interesting account of Lobachevsky's career as student, professor, and rector of the Kasan University, and an historical and critical discussion of his mathematical writings. Of particular interest is the chapter on the relation of Gauss, Lobachevsky, and Bolyai, and the conclusion reached by the author that each discovered the non-euclidean geometry independently of the others. Lobachevsky was connected with Gauss through his teacher Bartels who was a friend of Gauss, and from this fact it has been argued that Lobachevsky's discoveries were inspired by Gauss. Against this theory, Professor Engel presents facts to show that Lobachevsky could have learned from Bartels nothing more than that Gauss questioned the correctness of the euclidean axiom. Lobachevsky's own writings however do not bear out the hypothesis that he learned even this much from his teacher, for the gradual development of his thought is apparent in his unpublished works, from the time when in 1816 he attempted the proof of the disputed axiom, through the period when he recognized the failure of all attempts at such a 
proof but had not yet anything to take its place, until in 1826 he had found his way successfully through all his difficulties. He adds his own testimony as to the origin of his great theories in the opening sentences of the "Neue Anfangsgründe" in which he declares that the futility of the efforts made during two thousand years since Euclid to complete the theory of parallel lines aroused in him the suspicion that the ideas sought to be proved were not necessarily true. While it is remarkable that the solution of a two-thousand year old problem should be given almost simultaneously by three men, it should be remembered that these three were not the only mathematicians who had worked upon the problem. More than one had missed the solution by a hairsbreadth only; Lobachevsky, Bolyai, and Gauss succeeded in finding it.

Massachusetts Institute

Frederick S. Woods. OF TECHNOLOGY.

\section{VOGT'S ALGEBRAIC SOLUTION OF EQUATIONS.}

Leçons sur la Résolution algébrique des Équations. Par H. Vogt, professeur adjoint à la Faculté des Sciences de Nancy. Paris, Nony et Cie., 1895. 8vo., viii+201 pp.

The present work is, we suppose, intended to be an introduction to the modern theory of the algebraic solution of equations. It is true that the word modern does not appear in the title, but however elementary the character of a new book of this kind may be, it is natural to suppose that the author will present his material in accordance with modern points of view, as far as these are elementary and simple.

This, however, is not the case with the volume in hand, as we proceed to show. First and foremost we have the following serious criticism to make. The rockbed of the modern theory of the algebraic solution of equations is the principles of Galois. A text book on this subject which does not explain these with all detail and use them systematically from start to finish cannot be called modern.

That the present volume sins grievously in this respect can be shown at once. Galois' theory proposes a perfectly simple and uniform scheme for the solution of any given equation. In a work of this kind this scheme should be developed at the start and then undeviatingly employed 